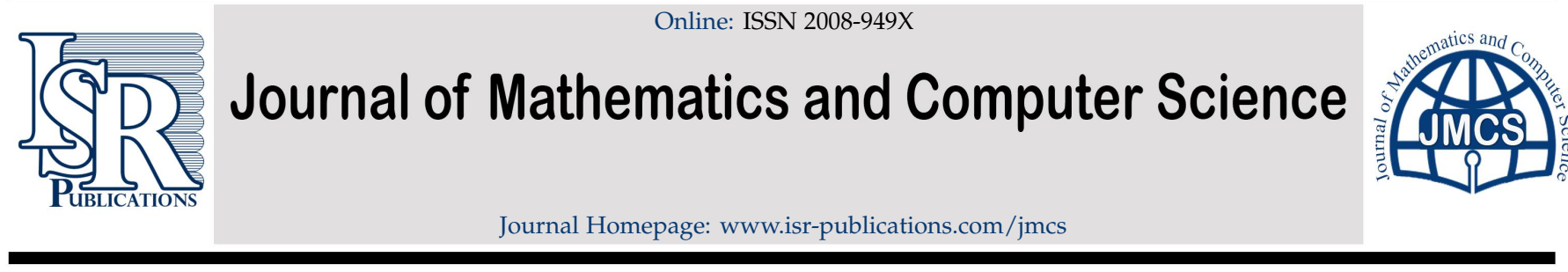

\title{
Modified differential transform scheme for solving systems of first order ordinary differential equations
}

\author{
S. Al-Ahmad, I. M. Sulaiman*, M. Mamat, Puspa Liza Ghazali
}

Faculty of Informatics and Computing, Universiti Sultan Zainal Abidin, Terengganu, Besut Campus, 22200, Malaysia.

\begin{abstract}
In this paper, a modified differential transform scheme (MDTS) is proposed for solving systems of first order ordinary differential equations. This numerical scheme serves as an alternative approach that is designed based on the differential transform method (DTM), Laplace transform and Padé approximants. The proposed method would be able to overcome the difficulty of solving these types of problems and also, gives a virtuous approximation for the true solution of the problems in a large region. Preliminary results are presented based on some examples which illustrate the validity and applicability of the proposed scheme. Also, all the obtained results corresponded to exact solutions.
\end{abstract}

Keywords: Systems of first order ordinary differential equations, differential transform method, Laplace transform, Padé approximants.

2020 MSC: 34A45, 44A10, 65L99.

(C)2021 All rights reserved.

\section{Introduction}

Consider the following system of ordinary differential equations of the first order

$$
\left\{\begin{array}{c}
y_{1}{ }^{\prime}=f_{1}\left(x, y_{1}, \ldots, y_{n}\right) \\
y_{2}{ }^{\prime}=f_{2}\left(x, y_{1}, \ldots, y_{n}\right) \\
\vdots \\
y_{n}{ }^{\prime}=f_{n}\left(x, y_{1}, \ldots, y_{n}\right),
\end{array}\right.
$$

where $y_{1}{ }^{\prime}, y_{2}{ }^{\prime}, \ldots, y_{n}{ }^{\prime}$ denotes the first derivative of the unknown functions and considered as a mapping relying on $x$, and $n$ unknown functions $f_{1} ; \ldots ; f_{n}$.

Since each equation of order $n$ can be written as a system consisting of $n$ ordinary differential equation of order one, then, the MDTS will be used without the need of linearization or smallness assumptions $[4,5,9]$. The differential transform method (DTM) which constructs an analytical solution in the form of a polynomial was first introduced by Zhou [19] as a new idea for obtaining the solution of differential

\footnotetext{
${ }^{*}$ Corresponding author

Email address: sulaimanib@unisza.edu.my (I. M. Sulaiman)

doi: $10.22436 /$ jmcs.022.01.07
}

Received: 2020-04-29 Revised: 2020-05-12 Accepted: 2020-05-16 
equations. This polynomials form is used as the approximation to exact solutions which are sufficiently differentiable. The idea of DTM is based on the concept of Taylor series [6, 11, 16], whose solution are usually obtained in series form. DTM is an iterative approach for obtaining Taylor series solutions of differential equations. Recently, the DTM has been extended to initial value problems, difference equations, and boundary value problems.

However, the major drawback of the DTM is obtaining a truncated series whose solution is a good approximation for the true solution of the problem in a very small region [15]. To overcome this drawback, the study proposed an alternative scheme to improve the accuracy of DTM by modifying the series solution of systems of first order ordinary differential equations. The proposed scheme starts by applying the Laplace transformation to the truncated series gotten by DTM, followed by employing the Padé approximants to convert the transformed series into a meromorphic function, and lastly obtaining an analytic solution using the inverse Laplace transform. This obtained solution may be an improved approximation or periodic solution compared to the DTM truncated series solution.

In this paper, Section 2 discusses brief overview and some fundamental results of DTM, Padé approximants and Laplace transform. We apply the new scheme to various examples to illustrates the simplicity and efficiency of the proposed scheme in Section 3. Lastly, the conclusion and discussion are in Section 4.

\section{Preliminaries}

This section presents some definitions of DTM and Padé approximants.

\subsection{Differential transform method}

Definition 2.1 ([2]). For a function $f(x)$ that is analytical at $x_{0}$ in the domain of interest, then

$$
F(k)=\frac{f^{(k)}\left(x_{0}\right)}{k !} .
$$

Definition 2.2 ([2]). The inverse differential transform is given as

$$
f(x)=\sum_{k=0}^{\infty} F(k)\left(x-x_{0}\right)^{k}
$$

Let the differential transforms of $u(x), g(x)$, and $h(x)$ be $U(k), G(k)$, and $H(k)$, respectively at $x_{0}=0$. Then the main operations of the DTM is presented in Table 1.

Table 1: Main operations of the DTM.

\begin{tabular}{|c|c|}
\hline Original function & Transformed function \\
\hline$u(x)=g(x)+h(x)$ & $U(k)=G(k)+H(k)$ \\
\hline$u(x)=c g(x)$ & $U(k)=c G(k)$ \\
\hline$u(x)=\frac{d^{n} g(x)}{d x^{n}}$ & $U(k)=\frac{(k+n) !}{k !} G(k+n)$ \\
\hline$u(x)=g(x) h(x)$ & $u(k)=\sum_{i=0}^{k} G(i) H(k-i)$ \\
\hline$u(x)=x^{n}$ & $U(k)=\delta(k-n)$ \\
\hline$u(x)=\exp (c x)$ & $U(k)=\frac{c^{k}}{k !}$ \\
\hline$u(x)=\cos (w x)$ & $u(k)=\frac{w^{k}}{k !} \cos \left(\frac{k \pi}{2}\right)$ \\
\hline$u(x)=\sin (w x)$ & $u(k)=\frac{w^{k}}{k !} \sin \left(\frac{k \pi}{2}\right)$ \\
\hline
\end{tabular}

Theorem 2.3 ([12]). If $f(y)=y^{m}$, then

$$
F(k)= \begin{cases}(Y(0))^{m}, & k=0, \\ \frac{1}{Y(0)} \sum_{r=1}^{k}\left(\frac{(m+1) r-k}{k}\right) Y(r) F(k-r), & k \geqslant 1 .\end{cases}
$$


The proof is obvious from the operations of differential transform that are given in Table 1 and Definitions 2.1 and 2.2.

A differential equation in the domain of interest can be transformed into an equation of algebraic form in the K-domain using differential transform, and the finite-term Taylor series expansion plus a remainder can further be applied to obtain $f(t)$ as

$$
f(t)=\sum_{k=0}^{N} F(k) \frac{\left(t-t_{0}\right)^{k}}{k !}+R_{N+1}(t) .
$$

The series solution (2.3) converges rapidly only in a small region. However, the convergence is often very slow in the wide region, and thus, yield an inaccurate truncations result.

In the MDTS, we start by applying the Laplace transformation to the truncated series gotten by DTM, followed by employing the Padé approximants to convert the transformed series into a meromorphic function, and lastly obtaining an analytic solution using the inverse Laplace transform. This obtained solution may be an improved approximation or periodic solution compared to the DTM truncated series solution.

For further reference on differential transform and DTM see [1-3, 12, 13, 17].

\subsection{Padé approximation}

The approximants of Padé refers to the ratio of two polynomials developed from Taylor series expansion coefficients the function $y(x)$. The $[\mathrm{L} / \mathrm{M}]$ Padé approximants to $\mathrm{y}(\mathrm{x})$ are defined by $[7,8]$

$$
\left[\frac{\mathrm{L}}{\mathrm{M}}\right]=\frac{\mathrm{P}_{\mathrm{L}}(\mathrm{x})}{\mathrm{Q}_{\mathrm{M}}(\mathrm{x})}
$$

and $P_{L}(x)$ and $Q_{M}(x)$ are known as the polynomials of degree at most $L$ and $M$, respectively. More so,

$$
\begin{gathered}
y(x)=\sum_{i=1}^{\infty} a_{i} x^{i}, \\
y(x)-\frac{P_{L}(x)}{Q_{M}(x)}=O\left(x^{L+M+1}\right),
\end{gathered}
$$

are the formal power series determining the coefficients of the polynomials $P_{L}(x)$ and $Q_{M}(x)$ by the equation. It is obvious the numerator and denominator of $[L / M]$ remain unchanged even after multiplying by a constant, thus, we can impose the normalization condition

$$
\mathrm{Q}_{M}(0)=1 \text {. }
$$

Lastly, $P_{L}(x)$ and $Q_{M}(x)$ are required to have no common factors. Rewriting the coefficient of $P_{L}(x)$ and $\mathrm{Q}_{M}(\mathrm{x})$ as

$$
\left\{\begin{array}{l}
P_{L}(x)=p_{0}+p_{1} x+p_{2} x^{2}+\cdots+p_{L} x^{L} \\
Q_{M}(x)=q_{0}+q_{1} x+q_{2} x^{2}+\cdots+q_{M} x^{M}
\end{array}\right.
$$

also, from (2.5) and (2.6), we can multiply (2.4) by $\mathrm{Q}_{M}(x)$, there by linearizing the coefficient equations. Rewriting (2.4) in more detailed form, gives

$$
\left\{\begin{array}{c}
a_{L+1}+a_{L} q_{1}+\cdots+a_{L-M+1} q_{M}=0 \\
a_{L+2}+a_{L+1} q_{1}+\cdots+a_{L-M+2} q_{M}=0 \\
\vdots \\
a_{L+M}+a_{L+M-1} q_{1}+\cdots+a_{L} q_{M}=0
\end{array}\right.
$$




$$
\left\{\begin{array}{l}
a_{0}=p_{0} \\
a_{0}+a_{0} q_{1}=p_{1}, \\
a_{2}+a_{1} q_{1}+a_{0} q_{2}=p_{2}, \\
\vdots \\
a_{L}+a_{L-1} q_{1}+\cdots+a_{0} q_{L}=p_{L} .
\end{array}\right.
$$

To obtain the solutions of the above equations, we begin with all the unknown $q^{\prime}$ s of the linear equations (2.7). Once we obtain the values of the $\mathrm{q}^{\prime} \mathrm{s}$, then (2.8) will produce an explicit formula for the all unknown $\mathrm{p}^{\prime} \mathrm{s}$, and thus, completes the solution.

If equation (2.7) and equation (2.8) are non-singular, then, their solution can be obtained directly as presented in equation (2.9), which implied that (2.9) holds, but if the index of the lower sum exceeds the upper, then, this sum would be replaced by zero:

$$
\left[\frac{L}{M}\right]=\frac{\operatorname{det}\left[\begin{array}{cccc}
a_{L-M+1} & a_{L-M+2} & \cdots & a_{L+1} \\
\cdot & \cdot & \cdot & \cdot \\
\cdot & \cdot & \cdot & \cdot \\
\sum_{j=M}^{L} a_{j-M} x^{j} & \sum_{j=M-1}^{L} a_{j-M+1} x^{j} & \cdots & \sum_{j=0}^{L} a_{j} x^{j}
\end{array}\right]}{\operatorname{det}\left[\begin{array}{cccc}
a_{L-M+1} & a_{L-M+2} & \cdots & a_{L+1} \\
\cdot & \cdot & \cdot & \cdot \\
\cdot & \cdot & \cdot & \cdot \\
\cdot & \cdot & \cdot & \cdot \\
a_{L} & a_{L+1} & \cdots & a_{L+M} \\
x^{M} & x^{M-1} & \cdots & 1
\end{array}\right]}
$$

From this step, the symbolic calculus software, MATLAB is often employed to obtain the Pade approximants diagonal matrix of orders including [2/2], [4/4] or [6/6].

Note that typically the Padé approximant, obtained from a partial Taylor sum, is more accurate than the latter. However; the Padé, being a rational expression, has poles, which are not present in the original function. It is a simple algebraic task to expand the form of an $[N, M]$ Padé in a Taylor series and compute the Padé coefficients by matching with the above [8].

\section{Numerical results}

This section presents the solutions of some examples for systems of first order ordinary differential equations.

Example 3.1. Consider the system of liner differential equations [14]

$$
\left\{\begin{array}{l}
y_{1}{ }^{\prime}(t)=y_{1}(t)+y_{2}(t), \\
y_{2}{ }^{\prime}(t)=-y_{1}(t)+y_{2}(t),
\end{array}\right.
$$

having initial conditions

$$
\left\{\begin{array}{l}
y_{1}(0)=0 \\
y_{2}(0)=1
\end{array}\right.
$$

and exact solution is $\left\{\begin{array}{l}y_{1}(t)=e^{t} \sin (t), \\ y_{2}(t)=e^{t} \cos (t) .\end{array}\right.$ Transforming Eq. (3.1) with conditions (3.2), gives

$$
\left\{\begin{array}{l}
(k+1) Y_{1}(k+1)=Y_{1}(k)+Y_{2}(k) \\
(k+1) Y_{2}(k+1)=-Y_{1}(k)+Y_{2}(k)
\end{array}\right.
$$


Thus,

$$
\left\{\begin{array}{l}
Y_{1}(k+1)=\frac{1}{k+1}\left[Y_{1}(k)+Y_{2}(k)\right] \\
Y_{2}(k+1)=\frac{1}{k+1}\left[-Y_{1}(k)+Y_{2}(k)\right]
\end{array}\right.
$$

and

$$
\left\{\begin{array}{l}
Y_{1}(0)=0 \\
Y_{2}(0)=1
\end{array}\right.
$$

Substituting Eq. (3.5) in Eq. (3.4), yields the values of $Y_{1}(k)$ and $Y_{2}(k)$ given in Table 2.

Table 2: The values of $Y_{1}(k)$ and $Y_{2}(k)$ for Example 3.1.

\begin{tabular}{|l|c|c|c|c|c|c|}
\hline $\mathrm{k}$ & 0 & 1 & 2 & 3 & 4 & 5 \\
\hline $\mathrm{Y}_{1}(\mathrm{k})$ & 0 & 1 & 1 & $\frac{1}{3}$ & 0 & $-\frac{1}{30}$ \\
\hline $\mathrm{Y}_{2}(\mathrm{k})$ & 1 & 1 & 0 & $-\frac{1}{3}$ & $-\frac{1}{6}$ & $-\frac{1}{30}$ \\
\hline
\end{tabular}

Using the inverse transformation rule 2.2, an approximate solution of (3.1) is obtained in the form

$$
\left\{\begin{array}{l}
y_{1}(t)=t+t^{2}+\frac{t^{3}}{3}-\frac{t^{5}}{30}+\ldots \\
y_{2}(t)=1+t-\frac{t^{3}}{3}-\frac{t^{4}}{6}-\frac{t^{5}}{30}+\ldots
\end{array}\right.
$$

which yields the exact solution of (3.1) in the limit of infinitely many terms.

To improve the accuracy of the differential transform solution (3.6), and to prove the power and efficiency for the MDTS, and by taking just until $\mathcal{O}\left(t^{5}\right)$ terms from (3.6), the MDTS is implemented as follows.

Applying the Laplace transform to the first $\mathcal{O}\left(t^{5}\right)$ terms from (3.6), yields

$$
\left\{\begin{array}{l}
\mathcal{L}\left(y_{1}(t)\right)=\frac{1}{s^{2}}+\frac{2}{s^{3}}+\frac{2}{s^{4}}-\frac{4}{s^{6}} \\
\mathcal{L}\left(y_{2}(t)\right)=\frac{1}{s}+\frac{1}{s^{2}}-\frac{2}{s^{4}}-\frac{4}{s^{5}}-\frac{4}{s^{6}}
\end{array}\right.
$$

For simplicity, let $s=\frac{1}{z}$, then

$$
\left\{\begin{array}{l}
\mathcal{L}\left(y_{1}(t)\right)=z^{2}+2 z^{3}+2 z^{4}-4 z^{6} \\
\mathcal{L}\left(y_{2}(t)\right)=z+z^{2}-2 z^{4}-4 z^{5}-4 z^{6}
\end{array}\right.
$$

The Padé approximants $\left[\frac{3}{3}\right]$ gives

$$
\left[\frac{3}{3}\right]=\left\{\begin{array}{l}
\mathcal{L}\left(y_{1}(t)\right)=\frac{z^{2}}{2 z^{2}-2 z+1} \\
\mathcal{L}\left(y_{2}(t)\right)=\frac{-z^{2}+z}{2 z^{2}-2 z+1}
\end{array}\right.
$$

Recalling $z=\frac{1}{s}$ to obtain $\left[\frac{3}{3}\right]$ in terms of $s$

$$
\left[\begin{array}{l}
3 \\
3
\end{array}\right]=\left\{\begin{array}{l}
\mathcal{L}\left(y_{1}(t)\right)=\frac{1}{s^{2}-2 s+2} \\
\mathcal{L}\left(y_{2}(t)\right)=\frac{s-1}{s^{2}-2 s+2}
\end{array}\right.
$$

Applying the inverse Laplace transform to the approximant $\left[\frac{3}{3}\right]$ of Padé, then, the modified approximate solution obtained is

$$
\left\{\begin{array}{l}
y_{1}(t)=e^{t} \sin (t) \\
y_{2}(t)=e^{t} \cos (t)
\end{array}\right.
$$

and this agrees with the exact solution for this problem. 
Example 3.2. Consider the following non-linear differential system [18]

$$
\left\{\begin{array}{l}
y_{1}{ }^{\prime}(t)+y_{2}{ }^{\prime}(t)+y_{1}(t)+y_{2}(t)=1 \\
y_{2}{ }^{\prime}(t)=2 y_{1}(t)+y_{2}(t)
\end{array}\right.
$$

with initial conditions

$$
\left\{\begin{array}{l}
y_{1}(0)=0 \\
y_{2}(0)=1
\end{array}\right.
$$

and exact solution is $\left\{\begin{array}{l}y_{1}(t)=e^{-t}-1, \\ y_{2}(t)=2-e^{-t} .\end{array}\right.$ Transforming Eq. (3.7) with conditions Eq. (3.8), produces

$$
\left\{\begin{array}{l}
(k+1) Y_{1}(k+1)+(k+1) Y_{2}(k+1)+Y_{1}(k)+Y_{2}(k)=\delta(k) \\
(k+1) Y_{2}(k+1)=2 Y_{1}(k)+Y_{2}(k) .
\end{array}\right.
$$

Thus,

$$
\left\{\begin{array}{l}
Y_{1}(k+1)=\frac{1}{k+1}\left[\delta(k)-(k+1) Y_{2}(k+1)-Y_{1}(k)-Y_{2}(k)\right] \\
Y_{2}(k+1)=\frac{1}{k+1}\left[2 Y_{1}(k)+Y_{2}(k)\right]
\end{array}\right.
$$

and

$$
\left\{\begin{array}{l}
Y_{1}(0)=0 \\
Y_{2}(0)=1
\end{array}\right.
$$

Substituting Eq. (3.10) in Eq. (3.9), produces the values of $Y_{1}(k)$ and $Y_{2}(k)$ shown in Table 3.

Table 3: The values of $Y_{1}(k)$ and $Y_{2}(k)$ for Example 3.2.

\begin{tabular}{|l|c|c|c|c|c|c|}
\hline $\mathrm{k}$ & 0 & 1 & 2 & 3 & 4 & 5 \\
\hline $\mathrm{Y}_{1}(\mathrm{k})$ & 0 & -1 & $\frac{1}{2}$ & $-\frac{1}{6}$ & $\frac{1}{24}$ & $-\frac{1}{120}$ \\
\hline $\mathrm{Y}_{2}(\mathrm{k})$ & 1 & 1 & $-\frac{1}{2}$ & $\frac{1}{6}$ & $-\frac{1}{24}$ & $\frac{1}{120}$ \\
\hline
\end{tabular}

Applying the inverse transformation rule 2.2, an approximate solution of (3.7) is obtained in the form

$$
\left\{\begin{array}{l}
y_{1}(t)=-t+\frac{t^{2}}{2}-\frac{t^{3}}{6}+\frac{t^{4}}{24}-\frac{t^{5}}{120}+\ldots \\
y_{2}(t)=1+t-\frac{t^{2}}{2}+\frac{t^{3}}{6}-\frac{t^{4}}{24}+\frac{t^{5}}{120}+\ldots
\end{array}\right.
$$

and this yields the exact solution of (3.7) in the limit of infinitely many terms. To improve the solution accuracy of the differential transform (3.11), and to prove the power and efficiency for the MDTS, and by taking just until $\mathcal{O}\left(\mathrm{t}^{5}\right)$ terms from (3.11), the MDTS is implemented as follows.

Applying the Laplace transform to the first $\mathcal{O}\left(t^{5}\right)$ terms from (3.11), yields

$$
\left\{\begin{array}{l}
\mathcal{L}\left(y_{1}(t)\right)=-\frac{1}{s^{2}}+\frac{1}{s^{3}}-\frac{1}{s^{4}}+\frac{1}{s^{5}}-\frac{1}{s^{6}} \\
\mathcal{L}\left(y_{2}(t)\right)=\frac{1}{s}+\frac{1}{s^{2}}-\frac{1}{s^{3}}+\frac{1}{s^{4}}-\frac{1}{s^{5}}+\frac{1}{s^{6}}
\end{array}\right.
$$

For simplicity, let $s=\frac{1}{z}$; then

$$
\left\{\begin{array}{l}
\mathcal{L}\left(y_{1}(t)\right)=-z^{2}+z^{3}-z^{4}+z^{5}-z^{6} \\
\mathcal{L}\left(y_{2}(t)\right)=z+z^{2}-z^{3}+z^{4}-z^{5}+z^{6}
\end{array}\right.
$$

The Padé approximants $\left[\frac{3}{3}\right]$ gives

$$
\left[\frac{3}{3}\right]=\left\{\begin{array}{l}
\mathcal{L}\left(y_{1}(t)\right)=-\frac{z^{2}}{z+1} \\
\mathcal{L}\left(y_{2}(t)\right)=\frac{2 z^{2}+z}{z+1}
\end{array}\right.
$$


Recalling $z=\frac{1}{s}$ to obtain $\left[\frac{3}{3}\right]$ in terms of $s$

$$
\left[\frac{3}{3}\right]=\left\{\begin{array}{l}
\mathcal{L}\left(y_{1}(t)\right)=-\frac{1}{s^{2}+s} \\
\mathcal{L}\left(y_{2}(t)\right)=\frac{s+2}{s^{2}+s}
\end{array}\right.
$$

Applying the inverse Laplace transform to the $\left[\frac{3}{3}\right]$ Padé approximant will produce the modified approximate solution

$$
\left\{\begin{array}{l}
y_{1}(t)=e^{-t}-1 \\
y_{2}(t)=2-e^{-t}
\end{array}\right.
$$

which agrees with the exact solution of this problem.

Example 3.3. Consider the system of non-homogeneous differential equations from [10]

$$
\left\{\begin{array}{l}
y_{1}{ }^{\prime}(t)=y_{3}(t)-\cos (t) \\
y_{2}^{\prime}(t)=y_{3}(t)-e^{t} \\
y_{3}{ }^{\prime}(t)=y_{1}(t)-y_{2}(t)
\end{array}\right.
$$

subject to the initial conditions

$$
\left\{\begin{array}{l}
y_{1}(0)=1 \\
y_{2}(0)=0 \\
y_{3}(0)=2
\end{array}\right.
$$

and exact solution $\left\{\begin{array}{l}y_{1}(t)=e^{t}, \\ y_{2}(t)=\sin (t), \\ y_{3}(t)=e^{t}+\cos (t) .\end{array}\right.$ Transforming Eq. (3.12) with conditions Eq. (3.13), we obtain

$$
v\left\{\begin{aligned}
(k+1) Y_{1}(k+1) & =Y_{3}(k)-\frac{1}{k !} \cos \left(\frac{k \pi}{2}\right) \\
(k+1) Y_{2}(k+1) & =Y_{3}(k)-\frac{1}{k !} \\
(k+1) Y_{3}(k+1) & =Y_{1}(k)-Y_{2}(k) .
\end{aligned}\right.
$$

Thus,

$$
\left\{\begin{array}{l}
Y_{1}(k+1)=\frac{1}{k+1}\left[Y_{3}(k)-\frac{1}{k !} \cos \left(\frac{k \pi}{2}\right)\right] \\
Y_{2}(k+1)=\frac{1}{k+1}\left[Y_{3}(k)-\frac{1}{k !}\right] \\
Y_{3}(k+1)=\frac{1}{k+1}\left[Y_{1}(k)-Y_{2}(k)\right]
\end{array}\right.
$$

and

$$
\left\{\begin{array}{l}
Y_{1}(0)=1 \\
Y_{2}(0)=0 \\
Y_{3}(0)=2
\end{array}\right.
$$

Substituting Eq. (3.16) in Eq. (3.15), produces the values of $Y_{1}(k), Y_{2}(k)$ and $Y_{3}(k)$ given in Table 4.

Table 4: The values of $Y_{1}(k), Y_{2}(k)$ and $Y_{3}(k)$ for Example 3.3.

\begin{tabular}{|l|c|c|c|c|c|c|}
\hline$k$ & 0 & 1 & 2 & 3 & 4 & 5 \\
\hline$Y_{1}(k)$ & 1 & 1 & $\frac{1}{2}$ & $\frac{1}{6}$ & $\frac{1}{24}$ & $\frac{1}{120}$ \\
\hline$Y_{2}(k)$ & 0 & 1 & 0 & $-\frac{1}{6}$ & 0 & $\frac{1}{120}$ \\
\hline$Y_{3}(k)$ & 2 & 1 & 0 & $\frac{1}{6}$ & $\frac{1}{12}$ & $\frac{1}{120}$ \\
\hline
\end{tabular}

Applying rule 2.2, an approximate solution of (3.12) is obtained in the form

$$
\left\{\begin{array}{l}
y_{1}(t)=1+t+\frac{t^{2}}{2}+\frac{t^{3}}{6}+\frac{t^{4}}{24}+\frac{t^{5}}{120}+\ldots \\
y_{2}(t)=t-\frac{t^{3}}{6}+\frac{t^{5}}{120}+\ldots \\
y_{3}(t)=2+t+\frac{t^{3}}{6}+\frac{t^{4}}{12}+\frac{t^{5}}{120}+\ldots
\end{array}\right.
$$


and this yields the exact solution of (3.12) in the limit of infinitely many terms. The MDTS is employed to improve the accuracy of (3.17), by taking just until $\mathcal{O}\left(t^{5}\right)$ terms from (3.17) as follows.

Applying the Laplace transform to the first $\mathcal{O}\left(t^{5}\right)$ terms from (3.17), yields

$$
\left\{\begin{array}{l}
\mathcal{L}\left(y_{1}(t)\right)=\frac{1}{s}+\frac{1}{s^{2}}+\frac{1}{s^{3}}+\frac{1}{s^{4}}+\frac{1}{s^{5}}+\frac{1}{s^{6}} \\
\mathcal{L}\left(y_{2}(t)\right)=\frac{1}{s^{2}}-\frac{1}{s^{4}}+\frac{1}{s^{6}} \\
\mathcal{L}\left(y_{3}(t)\right)=\frac{2}{s}+\frac{1}{s^{2}}+\frac{1}{s^{4}}+\frac{2}{s^{5}}+\frac{1}{s^{6}} .
\end{array}\right.
$$

For simplicity, let $\mathrm{s}=\frac{1}{z}$, then

$$
\left\{\begin{array}{l}
\mathcal{L}\left(y_{1}(t)\right)=z+z^{2}+z^{3}+z^{4}+z^{5}+z^{6} \\
\mathcal{L}\left(y_{2}(t)\right)=z^{2}-z^{4}+z^{6} \\
\mathcal{L}\left(y_{3}(t)\right)=2 z+z^{2}+z^{4}+2 z^{5}+z^{6}
\end{array}\right.
$$

The Padé approximants $\left[\frac{3}{3}\right]$ gives

$$
\left[\begin{array}{l}
3 \\
3
\end{array}\right]=\left\{\begin{array}{l}
\mathcal{L}\left(\mathrm{y}_{1}(\mathrm{t})\right)=-\frac{z}{z-1} \\
\mathcal{L}\left(\mathrm{y}_{2}(\mathrm{t})\right)=\frac{z^{2}}{z^{2}+1} \\
\mathcal{L}\left(\mathrm{y}_{3}(\mathrm{t})\right)=\frac{4 z^{3}+3 z^{2}-2 z}{z^{2}+2 z-1}
\end{array}\right.
$$

Recalling $z=\frac{1}{s}$ to obtain $\left[\frac{3}{3}\right]$ in terms of $s$

$$
\left[\frac{3}{3}\right]=\left\{\begin{array}{l}
\mathcal{L}\left(y_{1}(t)\right)=\frac{1}{s-1} \\
\mathcal{L}\left(y_{2}(t)\right)=\frac{1}{s^{2}+1} \\
\mathcal{L}\left(y_{3}(t)\right)=\frac{-2 s^{2}+s-1}{-s^{3}+s^{2}-s+1}
\end{array}\right.
$$

Applying the inverse Laplace transform to the $\left[\frac{3}{3}\right]$ Padé approximant, the modified approximate solution obtained is

$$
\left\{\begin{array}{l}
y_{1}(t)=e^{t} \\
y_{2}(t)=\sin (t), \\
y_{3}(t)=e^{t}+\cos (t),
\end{array}\right.
$$

and this agrees with the exact solution of this problem.

Example 3.4. Consider the non-linear system of differential equations [9]

$$
\left\{\begin{array}{l}
y_{1}^{\prime}(t)=2 y_{2}^{2}(t) \\
y_{2}^{\prime}(t)=e^{-t} y_{1}(t) \\
y_{3}^{\prime}(t)=y_{2}(t)+y_{3}(t)
\end{array}\right.
$$

subject to the initial conditions

$$
\left\{\begin{array}{l}
y_{1}(0)=1 \\
y_{2}(0)=1 \\
y_{3}(0)=0
\end{array}\right.
$$

and exact solution $\left\{\begin{array}{l}y_{1}(t)=e^{2 t}, \\ y_{2}(t)=e^{t}, \\ y_{3}(t)=t e^{t} .\end{array}\right.$ Transforming Eq. (3.18) with the conditions (3.19), gives

$$
\left\{\begin{array}{l}
(k+1) Y_{1}(k+1)=2 \sum_{i=0}^{k} Y_{2}(i) Y_{2}(k-i) \\
(k+1) Y_{2}(k+1)=\sum_{m=0}^{k} \frac{(-1)^{m}}{m !} Y_{1}(k-m) \\
(k+1) Y_{3}(k+1)=Y_{2}(k)+Y_{3}(k)
\end{array}\right.
$$


Thus,

$$
\left\{\begin{array}{l}
Y_{1}(k+1)=\frac{2}{k+1} \sum_{i=0}^{k} Y_{2}(i) Y_{2}(k-i) \\
Y_{2}(k+1)=\frac{1}{k+1} \sum_{m=0}^{k} \frac{(-1)^{m}}{m !} Y_{1}(k-m) \\
Y_{3}(k+1)=\frac{1}{k+1}\left[Y_{2}(k)+Y_{3}(k)\right]
\end{array}\right.
$$

and

$$
\left\{\begin{array}{l}
Y_{1}(0)=1 \\
Y_{2}(0)=1 \\
Y_{3}(0)=0
\end{array}\right.
$$

Substituting Eq. (3.21) in Eq. (3.20), produces the values of $Y_{1}(k), Y_{2}(k)$ and $Y_{3}(k)$ presented in Table 5.

Table 5: The values of $Y_{1}(k), Y_{2}(k)$ and $Y_{3}(k)$ for Example 3.4.

\begin{tabular}{|l|c|c|c|c|c|c|}
\hline $\mathrm{k}$ & 0 & 1 & 2 & 3 & 4 & 5 \\
\hline $\mathrm{Y}_{1}(\mathrm{k})$ & 1 & 2 & 2 & $\frac{4}{3}$ & $\frac{2}{3}$ & $\frac{4}{15}$ \\
\hline $\mathrm{Y}_{2}(\mathrm{k})$ & 1 & 1 & $\frac{1}{2}$ & $\frac{1}{6}$ & $\frac{1}{24}$ & $\frac{1}{120}$ \\
\hline $\mathrm{Y}_{3}(\mathrm{k})$ & 0 & 1 & 1 & $\frac{1}{2}$ & $\frac{1}{6}$ & $\frac{1}{24}$ \\
\hline
\end{tabular}

From Table 5, an approximate solution of (3.18) is obtained in the form

$$
\left\{\begin{array}{l}
y_{1}(t)=1+2 t+2 t^{2}+\frac{4}{3} t^{3}+\frac{2}{3} t^{4}+\frac{4}{15} t^{5}+\ldots \\
y_{2}(t)=1+t+\frac{t^{2}}{2}+\frac{t^{3}}{6}+\frac{t^{4}}{24}+\frac{t^{5}}{120}+ \\
y_{3}(t)=t+t^{2}+\frac{t^{3}}{2}+\frac{t^{4}}{6}+\frac{t^{5}}{24}+\ldots
\end{array}\right.
$$

which gives the exact solution of (3.18) in the limit of infinitely many terms. Next, the MDTS is employed to improve the accuracy of the solution (3.22). By taking just until $\mathcal{O}\left(\mathrm{t}^{5}\right)$ terms from (3.22), the MDTS is implemented as follows.

Applying the Laplace transform to the first $\mathcal{O}\left(t^{5}\right)$ terms from (3.22), yields

$$
\left\{\begin{array}{l}
\mathcal{L}\left(y_{1}(t)\right)=\frac{1}{s}+\frac{2}{s^{2}}+\frac{4}{s^{3}}+\frac{8}{s^{4}}+\frac{16}{s^{5}}+\frac{32}{s^{6}} \\
\mathcal{L}\left(y_{2}(t)\right)=\frac{1}{s}+\frac{1}{s^{2}}+\frac{1}{s^{3}}+\frac{1}{s^{4}}+\frac{1}{s^{5}}+\frac{1}{s^{6}} \\
\mathcal{L}\left(y_{3}(t)\right)=\frac{1}{s^{2}}+\frac{2}{s^{3}}+\frac{3}{s^{4}}+\frac{4}{s^{5}}+\frac{5}{s^{6}}
\end{array}\right.
$$

For simplicity, let $s=\frac{1}{z}$; then

$$
\left\{\begin{array}{l}
\mathcal{L}\left(\mathrm{y}_{1}(\mathrm{t})\right)=z+2 z^{2}+4 z^{3}+8 z^{4}+16 z^{5}+32 z^{6} \\
\mathcal{L}\left(\mathrm{y}_{2}(\mathrm{t})\right)=z+z^{2}+z^{3}+z^{4}+z^{5}+z^{6} \\
\mathcal{L}\left(\mathrm{y}_{3}(\mathrm{t})\right)=z^{2}+2 z^{3}+3 z^{4}+4 z^{5}+5 z^{6}
\end{array}\right.
$$

The Padé approximants $\left[\frac{3}{3}\right]$ give

$$
\left[\frac{3}{3}\right]=\left\{\begin{array}{l}
\mathcal{L}\left(y_{1}(t)\right)=-\frac{z}{2 z-1} \\
\mathcal{L}\left(y_{2}(t)\right)=-\frac{z}{z-1} \\
\mathcal{L}\left(y_{3}(t)\right)=\frac{z^{2}}{z^{2}-2 z+1}
\end{array}\right.
$$

Recalling $z=\frac{1}{s}$ to obtain $\left[\frac{3}{3}\right]$ in terms of $s$

$$
\left[\begin{array}{l}
3 \\
3
\end{array}\right]=\left\{\begin{array}{l}
\mathcal{L}\left(y_{1}(t)\right)=\frac{1}{s-2} \\
\mathcal{L}\left(y_{2}(t)\right)=\frac{1}{s-1} \\
\mathcal{L}\left(y_{3}(t)\right)=\frac{1}{s^{2}-2 s+1}
\end{array}\right.
$$


Applying the inverse Laplace transform to the $\left[\frac{3}{3}\right]$ Padé approximant, the modified approximate solution obtained is

$$
\left\{\begin{array}{l}
y_{1}(t)=e^{2 t}, \\
y_{2}(t)=e^{t}, \\
y_{3}(t)=t e^{t},
\end{array}\right.
$$

which is excellent agreement with the exact solution for this example.

Example 3.5. Consider the nonlinear system of differential equations [14]

$$
\left\{\begin{array}{l}
y_{1}^{\prime}(t)=2 e^{4 t} y_{4}{ }^{2}(t) \\
y_{2}^{\prime}(t)=y_{1}(t)-y_{3}(t)+\cos (t)-e^{2 t} \\
y_{3}^{\prime}(t)=y_{2}(t)-y_{4}(t)+e^{-t}-\sin (t) \\
y_{4}^{\prime}(t)=-e^{-5 t} y_{1}{ }^{\prime}(t)
\end{array}\right.
$$

subject to the initial conditions

$$
\begin{cases}y_{1}(0)=1, & y_{2}(0)=1 \\ y_{3}(0)=0, & y_{4}(0)=1\end{cases}
$$

and exact solution is $\left\{\begin{array}{l}y_{1}(t)=e^{2 t} \\ y_{2}(t)=\sin (t)+\cos (t), \\ y_{3}(t)=\sin (t), \\ y_{4}(t)=e^{-t}\end{array}\right.$

Transforming Eq. (3.23) with the conditions Eq. (3.24), gives

$$
\left\{\begin{array}{l}
(k+1) Y_{1}(k+1)=2 \sum_{i=0}^{k} \frac{4^{i}}{i !} G(k-i) \\
(k+1) Y_{2}(k+1)=Y_{1}(k)-Y_{3}(k)+\frac{1}{k !} \cos \left(\frac{k \pi}{2}\right)-\frac{2^{k}}{k !} \\
(k+1) Y_{3}(k+1)=Y_{2}(k)-Y_{4}(k)+\frac{(-1)^{k}}{k !}-\frac{1}{k !} \sin \left(\frac{k \pi}{2}\right) \\
(k+1) Y_{4}(k+1)=-\sum_{m=0}^{k} \frac{(-5)^{m}}{m !} H(k-m) .
\end{array}\right.
$$

Thus,

$$
\left\{\begin{array}{l}
Y_{1}(k+1)=\frac{2}{k+1} \sum_{i=0}^{k} \frac{4^{i}}{i !} G(k-i) \\
Y_{2}(k+1)=\frac{1}{k+1}\left[Y_{1}(k)-Y_{3}(k)+\frac{1}{k !} \cos \left(\frac{k \pi}{2}\right)-\frac{2^{k}}{k !}\right] \\
Y_{3}(k+1)=\frac{1}{k+1}\left[Y_{2}(k)-Y_{4}(k)+\frac{(-1)^{k}}{k !}-\frac{1}{k !} \sin \left(\frac{k \pi}{2}\right)\right] \\
Y_{4}(k+1)=-\frac{1}{k+1} \sum_{m=0}^{k} \frac{(-5)^{m}}{m !} H(k-m)
\end{array}\right.
$$

and

$$
\begin{cases}Y_{1}(0)=1, & Y_{2}(0)=1 \\ Y_{3}(0)=0, & Y_{4}(0)=1,\end{cases}
$$

where $G(k)$ and $H(k)$ are the differential transform of $g\left(y_{4}\right)=y_{4}{ }^{2}$ and $h\left(y_{1}\right)=y_{1}{ }^{2}$, respectively.

By Theorem 2.3, the differential transform $\mathrm{G}(\mathrm{k}), \mathrm{H}(\mathrm{k})$ in Eq. (3.25) will be

$$
\begin{aligned}
& G(0)=\left(Y_{4}(0)\right)^{2}=1, \\
& G(k)=\sum_{r=1}^{k}\left(\frac{3 r-k}{k}\right) Y_{4}(r) G(k-r), k \geqslant 1,
\end{aligned}
$$

and

$$
\begin{aligned}
& H(0)=\left(Y_{1}(0)\right)^{2}=1 \\
& H(k)=\sum_{r=1}^{k}\left(\frac{3 r-k}{k}\right) Y_{1}(r) H(k-r), \quad k \geqslant 1 .
\end{aligned}
$$


Substituting Eq. (3.26), Eq. (3.27), and Eq. (3.28) in Eq. (3.25), we get

$$
\left\{\begin{array}{l}
Y_{1}(1)=2, \quad Y_{2}(1)=1, \\
Y_{3}(1)=1, \quad Y_{4}(1)=-1,
\end{array}\right.
$$

consequently, yields the following values of $Y_{1}(k), Y_{2}(k), Y_{3}(k)$ and $Y_{4}(k)$ which are shown in Table 6.

Table 6: The values of $Y_{1}(k), Y_{2}(k), Y_{2}(k)$ and $Y_{4}(k)$ for Example 3.5.

\begin{tabular}{|l|c|c|c|c|c|c|}
\hline$k$ & 0 & 1 & 2 & 3 & 4 & 5 \\
\hline$Y_{1}(k)$ & 1 & 2 & 2 & $\frac{4}{3}$ & $\frac{2}{3}$ & $\frac{4}{15}$ \\
\hline$Y_{2}(k)$ & 1 & 1 & $-\frac{1}{2}$ & $-\frac{1}{6}$ & $\frac{1}{24}$ & $-\frac{1}{120}$ \\
\hline$Y_{3}(k)$ & 0 & 1 & 0 & $-\frac{1}{6}$ & 0 & $\frac{1}{120}$ \\
\hline$Y_{4}(k)$ & 1 & -1 & $\frac{1}{2}$ & $-\frac{1}{6}$ & $\frac{1}{24}$ & $-\frac{1}{120}$ \\
\hline
\end{tabular}

Applying the inverse transformation rule 2.2, an approximate solution of (3.23) is obtained in the form

$$
\left\{\begin{array}{l}
y_{1}(t)=1+2 t+2 t^{2}+\frac{4}{3} t^{3}+\frac{2}{3} t^{4}+\frac{4}{15} t^{5}+\ldots, \\
y_{2}(t)=1+t-\frac{t^{2}}{2}-\frac{t^{3}}{6}+\frac{t^{4}}{24}-\frac{t^{5}}{120}+\ldots, \\
y_{3}(t)=t-\frac{t^{3}}{6}+\frac{t^{5}}{120}+\ldots, \\
y_{4}(t)=1-t+\frac{t^{2}}{2}-\frac{t^{3}}{6}+\frac{t^{4}}{24}-\frac{t^{5}}{120}+\ldots,
\end{array}\right.
$$

which gives the exact solution of (3.23) in the limit of infinitely many terms. From this point, we apply the MDTS to improve the accuracy of the differential transform solution (3.29). By taking just $\mathcal{O}\left(t^{5}\right)$ terms from (3.29), the MDTS is implemented as follows.

Applying the Laplace transform to the first $\mathcal{O}\left(t^{5}\right)$ terms from (3.29), yields

$$
\left\{\begin{array}{l}
\mathcal{L}\left(y_{1}(t)\right)=\frac{1}{s}+\frac{2}{s^{2}}+\frac{4}{s^{3}}+\frac{8}{s^{4}}+\frac{16}{s^{5}}+\frac{32}{s^{6}} \\
\mathcal{L}\left(y_{2}(t)\right)=\frac{1}{s}+\frac{1}{s^{2}}-\frac{1}{s^{3}}-\frac{1}{s^{4}}+\frac{1}{s^{5}}-\frac{1}{s^{6}} \\
\mathcal{L}\left(y_{3}(t)\right)=\frac{1}{s^{2}}-\frac{1}{s^{4}}+\frac{1}{s^{6}} \\
\mathcal{L}\left(y_{4}(t)\right)=\frac{1}{s}-\frac{1}{s^{2}}+\frac{1}{s^{3}}-\frac{1}{s^{4}}+\frac{1}{s^{5}}-\frac{1}{s^{6}}
\end{array}\right.
$$

For simplicity, let $s=\frac{1}{z}$, then

$$
\left\{\begin{array}{l}
\mathcal{L}\left(y_{1}(t)\right)=z+2 z^{2}+4 z^{3}+8 z^{4}+16 z^{5}+32 z^{6}, \mathcal{L}\left(y_{2}(t)\right)=z+z^{2}-z^{3}-z^{4}+z^{5}-z^{6} \\
\mathcal{L}\left(y_{3}(t)\right)=z^{2}-z^{4}+z^{6} \\
\mathcal{L}\left(y_{4}(t)\right)=z-z^{2}+z^{3}-z^{4}+z^{5}-z^{6}
\end{array}\right.
$$

The Padé approximants $\left[\frac{3}{3}\right]$ gives

$$
\left[\frac{3}{3}\right]=\left\{\begin{array}{l}
\mathcal{L}\left(y_{1}(t)\right)=-\frac{z}{2 z-1} \\
\mathcal{L}\left(y_{2}(t)\right)=\frac{z^{2}+z}{z^{2}+1} \\
\mathcal{L}\left(y_{3}(t)\right)=\frac{z^{2}}{z^{2}+1} \\
\mathcal{L}\left(y_{4}(t)\right)=\frac{z}{z+1}
\end{array}\right.
$$

Recalling $z=\frac{1}{s}$ to obtain $\left[\frac{3}{3}\right]$ in terms of $s$

$$
\left[\frac{3}{3}\right]=\left\{\begin{array}{l}
\mathcal{L}\left(y_{1}(t)\right)=\frac{1}{s-2} \\
\mathcal{L}\left(y_{2}(t)\right)=\frac{s+1}{s^{2}+1} \\
\mathcal{L}\left(y_{3}(t)\right)=\frac{1}{s^{2}+1} \\
\mathcal{L}\left(y_{4}(t)\right)=\frac{1}{s+1}
\end{array}\right.
$$


Applying the inverse Laplace transform to the $\left[\frac{3}{3}\right]$ Padé approximant, the modified approximate solution is

$$
\left\{\begin{array}{l}
y_{1}(t)=e^{2 t} \\
y_{2}(t)=\sin (t)+\cos (t) \\
y_{3}(t)=\sin (t), \\
y_{4}(t)=e^{-t},
\end{array}\right.
$$

which agrees with the exact solution for this example.

\section{Conclusion and discussion}

The MDTS is an efficient method for obtaining the approximate solutions for systems of first order differential equations. All the preliminary results obtained had shown that the proposed scheme is in excellent agreement with all results of the exact solution. These examples illustrate that the modified differential transform has significantly improves convergence rate of the truncated series solution obtained by DTM, and frequently produce the true analytic solution. We show that the proposed scheme is a very promising and can find wider applications in other fields.

\section{References}

[1] S. Al-Ahmad, M. Mamat, R. Al-Ahmad, Finding Differential Transform Using Difference Equations, IAENG Int. J. Appl. Math., 50 (2020), 127-132. 2.1

[2] S. Al-Ahmad, M. Mamat, R. Al-Ahmad, I. M. Sulaiman, P. L. Ghazali, M. A. Mohamed, On New Properties of Differential Transform via Difference Equations, Int. J. Eng. Tech., 7 (2018), 321-324. 2.1, 2.2

[3] A. H. Ali, Applications of Differential Transform Method To Initial Value Problems, Amer. J. Eng. Res., 6 (2017), $365-371$. 2.1

[4] A. K. Alomari, N. Ratib Anakira, A. S. Bataineh, I. Hashim, Approximate solution of nonlinear system of BVP arising in fluid flow problem, Math. Probl. Eng., 2013 (2013), 7 pages. 1

[5] N. Anakira, Solution of system of ordinary differential equations by optimal homotopy asymptotic method, AIP Conference Proceedings, 2096 (2019), 5 pages. 1

[6] F. Ayaz, Solutions of the system of differential equations by differential transform method, Appl. Math. Comput., 147 (2004), 547-567. 1

[7] G. A. Baker, Essentials of Padé approximants, Academic Press, London, (1975). 2.2

[8] C. M. Bender, S. A. Orszag, Advanced mathematical methods for scientists and engineers I: Asymptotic methods and perturbation theory, Springer, New York, (2013). 2.2, 2.2

[9] J. Biazar, E. Babolian, R. Islam, Solution of the system of ordinary differential equations by Adomian decomposition method, Appl. Math. Comput., 147 (2004), 713-719. 1, 3.4

[10] J. Biazar, H. Ghazvini, He's variational iteration method for solving linear and non-linear systems of ordinary differential equations, Appl. Math. Comput., 191 (2007), 287-297. 3.3

[11] C.-L. Chen, S.-H. Lin, C.-K. Chen, Application of Taylor transformation to nonlinear predictive control problem, Appl. Math. Modelling, 20 (1996), 699-710. 1

[12] B. Ibis, Approximate analytical solutions for nonlinear Emden-Fowler type equations by differential transform method, arXiv, 2012 (2012), 7 pages. 2.3, 2.1

[13] A. F. Jameel, N. R. Anakira, M. M. Rashidi, A. K. Alomari, A. Saaban, M. A. Shakhatreh, Differential Transformation Method For Solving High Order Fuzzy Initial Value Problems, Ital. J. Pure Appl. Math., 39 (2018), 194-208. 2.1

[14] F. Mirzaee, Differential Transform Method for Solving Linear and Nonlinear Systems of Ordinary Differential Equations, Appl. Math. Sci. (Ruse), 5 (2011), 3465-3472. 3.1, 3.5

[15] S. Momani, V. S. Ertúrk, Solutions of non-linear oscillators by the modified differential transform method, Comput. Math. Appl., 55 (2008), 833-842. 1

[16] Z. M. Odibat, Differential transform method for solvin Volterra integral equation with separable kernels, Math. Comput. Modelling, 48 (2008), 1144-1149. 1

[17] A. S. V. Ravi Kanth, K. Aruna, Differential transform method for solving linear and non-linear systems of partial differential equations, Phys. Lett. A, 372 (2008), 6896-6898. 2.1

[18] M. Thongmoon, S. Pusjuso, The numerical solutions of differential transform method and the Laplace transform method for a system of differential equations, Nonlinear Anal. Hybrid Syst., 4 (2010), 425-431. 3.2

[19] J. K. Zhou, Differential transformation and Application for electrical circuits, Huazhong University Press, Wuhan, (1986). 1 